\title{
Fortbildung
}

\section{MULTIPLE SKLEROSE BEI KINDERN UND JUGENDLICHEN - NEUE THERAPIEN UND WICHTIGE DIFFERENTIALDIAGNOSEN}

\section{Einleitung}

Die pädiatrische multiple Sklerose (MS) gehört zu den erworbenen chronisch entzündlichen ZNS-Erkrankungen des Kindes- und Jugendalters. Pathophysiologisch kommt es durch eine Störung der Blut-HirnSchranke zu einer Invasion inflammatorischer Zellen und Zytokine, die zu lokalen Entzündungsherden, Myelinödem und Myelinalteration führen ${ }^{1)}$. Mit einer Inzidenz von 0.07-2.9/100í000 gehört die pädiatrische MS - im Gegensatz zur adulten MS - zu den seltenen Erkrankungen ${ }^{2)}$. Weitere markante Unterschiede zur adulten MS sind die hohe Schubfrequenz und die Neigung zu infratentoriellen Läsionen zu Beginn der Erkrankung, die frühen kognitiven Defizite und Hirnatrophie sowie das Auftreten einer alltagsrelevanten körperlichen Einschränkung im Alter von ca. 35 Jahren bei unbehandelten Patienten ${ }^{3)}$. Für das Krankheitsverständnis essentiell ist die Tatsache, dass der chronisch-entzündliche Prozess sich in einem noch in Entwicklung befindenden Gehirn abspielt. Das ausgeprägt gute Erholungspotential von den ersten Schüben ist daher trügerisch ${ }^{4}$. In den letzten Jahren ist die Erkenntnis gewachsen, dass die pädiatrische MS alles andere als eine benigne Variante darstellt und eine Immuntherapie eingeleitet werden sollte, sobald die Diagnose definitiv steht. Hierfür sind eine akkurate, zeitnahe Diagnose unter Berücksichtigung möglicher Differentialdiagnosen und die Wahl des geeigneten Medikamentes essentiell. Da das Vorkommen der MS im Kindes- und Jugendalter erst in den letzten 10-15 Jahren so richtig ins Bewusstsein eingedrungen ist und man lange von der Annahme ausging, dass die pädiatrische MS milder verläuft, fehlen wichtige epidemiologische Daten zu Häufigkeit, Verläufen unter Therapie, Lebensqualität und Auswirkungen der Erkrankung auf die Alltagsaktivitäten der Kinder und Jugendlichen. Diese Wissenslücke soll nun mit Hilfe eines nationalen Registers geschlossen werden https://www.swiss-ped-ibraind.ch/.

\section{Diagnose pädiatrische MS}

Die Diagnose der pädiatrischen MS beruht ebenfalls auf dem Nachweis der zeitlichen und räumlichen Dissemination, wie sie in den aktuellen 2017 McDonald Kriterien ausgeführt werden ${ }^{5}$. Konkret versteht man unter der räumlichen Dissemination den Nachweis von demyelinisierenden Läsionen in mindestens 2 definierten Regionen (periventikulär, (juxta)kortikal, infratentoriell, spinal). Eine zeitliche Dissemination ist gegeben, wenn in einem MRI sowohl Kontrastmittel aufnehmende als auch nicht Kontrastmittel aufnehmende Läsionen vorhanden sind. Wenn das im ersten MRI nicht der Fall ist, gelten der Nachweis oligoklonaler Banden im Liquor oder eine neue, MS-typische Läsion in einem Verlaufs-MRI ebenfalls als Kriterien der zeitlichen Dissemination. Der Verlauf der pädiatrischen MS ist schubförmig-remittierend. Ein Schub ist definiert als neues fokal-neurologisches Defizit, das mindestens 24h kontinuierlich anhält. Schleichende Verschlechterungen ohne eindeutig abgrenzbare Schübe, wie sie im Erwachsenenalter bei der primär progredienten MS vorkommen, sind im Kindes- und Jugendalter hochverdächtig für eine genetisch-metabolische Erkrankung und nicht für eine pädiatrische MS. Initialsymptome beim ersten Schub sind heterogen. Während beim Jugendlichen die klassische Optikusneuritis häufig ist, dominieren beim jüngeren Kind polyfokale neurologische Ausfälle, oftmals mit grossflächigen, unscharf begrenzten Läsionen im MRI einhergehend ${ }^{6}$. Weitere häufige Symptome sind Ataxie, Vertigo und Störungen der Okulomotorik. Eine isolierte transverse Myelitis ist dagegen nur in ca. 13\% das Initialsymptom einer pädiatrischen MS. Da es weder eine eindeutige Ursache noch spezifische Biomarker gibt, bleibt die Diagnose der pädiatrischen MS nach sorgfältiger klinischer Untersuchung, Bildgebung und laborchemischer Abklärungen inklusive Lumbalpunktion eine Ausschlussdiagnose. Die Kenntnis des gesamten Spektrums der entzündlichen ZNS-Erkrankungen im Kindes- und Jugendalter sind daher für die Diagnose der pädiatrischen MS unabdingbar.

\section{Differentialdiagnose}

Bei Auftreten eines akuten fokalen neurologischen Defizits verbunden mit dem Nachweis von Läsionen der weissen Substanz im MRI wird in der Regel ein erstes akutes demyeliniserendes Ereignis (ADE) diagnostiziert. Neben der MS müssen in erster Linie eine Myelin-Oligodendrozyten Glykoprotein (MOG)-Antikörper assoziierte Erkrankung (MOGAD) und eine Aquaporin 4-Antikörper positive Neuromyelitis optica Spektrum Erkrankung (AQP4-NMOSD) in Betracht gezogen werden. Die Kenntnis der typischen klinischen Präsentation, wegweisender Laborparameter und Läsionsmuster im MRI (Tab. 1, Abb. 1) sowie der MS-Mimics ermöglichen in der Regel eine rasche Diagnosestellung. ADEs bei Kindern sind in mehr als der Hälfte der Fälle monophasische Ereignisse, die keiner Dauertherapie bedürfen ${ }^{7}$.

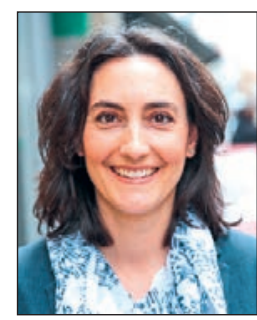

Sandra Bigi

https://doi.org/ $10.35190 / \mathrm{d} 2021.3 .5$

Korrespondenz sandra.bigi@insel.ch 


\section{Fortbildung}

Die Symptome der ADEs sind abhängig vom Ort der Läsion. Im Kindesalter sind das überwiegend:

1. Optikusneuritiden mit subakut auftretender Visusminderung und Bulbus-Bewegungsschmerz der betroffenen Seite.

2. Transverse Myelitis mit Querschnittssymptomatik und Schmerzen.

3. Infratentorielle Läsionen mit Ataxie, Störungen der Okulomotorik, sensiblen oder motorischen Defiziten.

4. ADEM (akute disseminierte Enzephalomyelitis) mit Enzephalopathie und polyfokalen neurologischen Defiziten.

\section{MOG-Antikörper assoziierte Erkrankung (MOGAD)}

Das Myelin Oligodendrozyten Glykoprotein (MOG) wird ausschliesslich auf Oligodendrozyten im ZNS exprimiert ${ }^{8)}$. MOG-Antikörper wurden initial bei Kindern mit ADEM beschrieben. Aktuelle Daten zeigen, dass sie bei etwa 30\% der Kinder mit ADEs nachgewiesen werden können, in der Mehrzahl der Fälle transient, und meistens mit einer monophasischen Erkrankung einhergehend $^{7}$. Die klinische Präsentation zeigt eine Altersabhängigkeit: Während bei jungen Kindern eine Präsentation mit ADEM überwiegt, entwickeln Kinder >9 Jahre häufiger einen «optikospinalen Phänotyp» mit Optikus-Neuritis, Myelitis oder Hirnstamm-Enzephalitis. Optikusneuritiden treten häufig bilateral auf und sind meistens langstreckig inkl. des vorderen Anteils der Sehnerven mit Beteiligung der Papille ${ }^{9}$. Die Höhe des MOG Antikörper-Titers korreliert nicht mit dem Schweregrad der Manifestation, möglicherweise aber der Titer-Verlauf mit dem Rezidivrisiko. Hohe MOG Antikörper-Titer schliessen eine MS praktisch aus. Es kommen auch Fälle mit multiplen Rezidiven vor, die noch nach Jahren auftreten können. Der Outcome ist häufig gut, Risikofaktoren für den Erwerb eines chronischen Defizits sind eine Manifestation mit tranverser Myelitis, sowie wahrscheinlich auch eine hohe Anzahl von Rezidiven ${ }^{10}$.

\section{AQP4-Antikörper positive Neuromyelitis} optica Spektrum Erkrankung (AQP4-NMOSD) Aquaporin4 (AQP4), ein Transmembranprotein, ist der häufigste Wasserkanal im ZNS. Er wird reichlich auf den Astrozyten, an den Fortsätzen an der Blut-HirnSchranke exprimiert. AQP4-Ak wurden zunächst bei erwachsenen Patienten mit Neuromyelitis optica (NMO) beschrieben. Die AQP4-NMOSD ist im Kindesalter selten, der Verlauf häufig schwerwiegend mit Rezidiven in etwa $80 \%$ der Fälle ${ }^{11}$. Die Rückbildung der Symptome nach einem akuten Schub ist häufig unvollständig. Auch Kinder haben ein hohes Risiko, eine alltagsrelevante Einschränkung zu erwerben, insbesondere eine Visusminderung nach Optikus-Neuritis und eine Beeinträchtigung der Blasen- und Mastdarm-Funktion ggf. kombiniert mit einer in der Regel spastischen Paraparese nach transverser Myelitis ${ }^{10}$. Charakteristisch für die AQP4-NMOSD kann zudem eine Beteiligung der Area postrema im Hirnstamm klinisch oftmals mit unstillbarem Erbrechen und/oder Singultus einhergehend - sowie auch diencephaler Strukturen sein. (Tabelle 1)

\section{Radiologische MS-Mimics und «Red flags»} Einer MS ähnliche MRI-Läsionen können bei einer Vielzahl von ZNS-Erkrankungen vorkommen, wie zum Beispiel bei Vaskulitiden, der Sarkoidose, einigen Leukodystrophien und der hämophagozytierenden Lymphohistiozytose ${ }^{12}$. Nicht korrekte neuroradiologische Einschätzungen sind zudem in einigen Fällen von M. Fabry, einer lysosomalen Speichererkrankung, beschrieben. Diese kann zu einer Angiopathie kleiner und grosser Gefässe mit Läsionen der weissen Substanz führen. Für eine MS im Kindesalter sind folgende klinische Zeichen untypisch (《red flags»): Ein langsam progredienter Verlauf, eine fehlende Erholung zumindest von den ersten Schüben, ein perakutes Auftreten von Symptomen innerhalb von Sekunden-Minuten (eher vaskulär), anhaltendes Fieber und Gewichtsabnahme, neues Auftreten epileptischer Anfälle, eine Schwerhörigkeit/Taubheit sowie eine Psychose.

\section{Therapie der pädiatrischen MS}

Entgegen der früheren Meinung, dass die pädiatrische MS weniger schlimm ist als im Erwachsenenalter, weil sich die jungen Patienten besser von den Schüben erholen, zeigen die Ergebnisse der Forschung das Gegenteil. Basierend auf den Erkenntnissen der letzten Jahre gibt es keinen Grund, Kindern und Jugendlichen mit MS eine Therapie vorzuenthalten. Es wird zwischen der akuten Schubtherapie und der längerfristigen Immuntherapie unterschieden.

Schubtherapie: Ziel der Schubtherapie ist eine Verkürzung der Schubdauer und raschere Erholung der Schubsymptome. Die Schubtherapie hat aber per se keinen Einfluss auf den zugrundeliegenden chronisch-entzündlichen Prozess, der - oftmals im Hintergrund - im ZNS abläuft. Hochdosiertes Methylprednisolon i.v. stellt die first-line Therapie bei Schüben dar. Die Dosierung beträgt idR 20-30 mg/kg/d für 3-5 Tage. Bei unzureichender Erholung sollte die Umstellung auf eine second-line Therapie evaluiert werden. Bei der pädiatrischen MS kann entweder die Methylprednisolontherapie 14 Tage nach Erstbehandlung wiederholt werden oder eine Umstellung auf eine Plasmapherese erfolgen. Die Plasmapherese sollte insbesondere bei persistierend schweren neurologischen Ausfällen oder bei fulminanten Schüben zum Zuge kommen.

Immuntherapie (Langzeittherapie): Das mittelfristige Ziel der Immuntherapie ist Schubfreiheit und Verhinderung neuer Hirnläsionen. Mit den zur Verfügung stehenden neueren MS-Therapeutika ist dieses Ziel bei fast allen pädiatrischen MS Patienten gut erreichbar. Das längerfristige Ziel besteht darin, kognitive Defizite und eine Hirnatrophie weitestgehend zu vermeiden bzw. das Fortschreiten aufzuhalten. Wie gut dies mit den aktuell verfügbaren Therapien möglich ist, ist aktuell Gegenstand der Forschung - hierzu sind die Langzeitdaten noch nicht verfügbar. Die vor- 


\section{Fortbildung}

Klinische Merkmale

\begin{tabular}{|c|c|c|c|}
\hline Weiblich:männlich & 1.8:1.0 & $1.0: 1.0$ & $4.0: 1.0$ \\
\hline Symptome < 11 Jahre & Selten & Häufig & Sehr selten \\
\hline \multicolumn{4}{|l|}{ Klinische Phänotypen } \\
\hline Optikus Neuritis & Häufig, monokular & $\begin{array}{l}\text { Häufig, oft bilateral, } \\
\text { häufig Papillitis }\end{array}$ & $\begin{array}{l}\text { Häufig, oft bilateral, häufig } \\
\text { Beteiligung des Chiasmas }\end{array}$ \\
\hline Transverse Myelitis & $\begin{array}{l}\text { Variabel, zum Teil klinisch } \\
\text { silent, meistens gute Erholung }\end{array}$ & $\begin{array}{l}\text { Häufig, zum Teil milde Symp- } \\
\text { tome, häufig Beteiligung } \\
\text { des Conus medullaris }\end{array}$ & $\begin{array}{l}\text { Häufig, typischerweise ausge- } \\
\text { prägte Defizite mit hohem } \\
\text { Risiko einer Persistenz }\end{array}$ \\
\hline $\begin{array}{l}\text { Häufige Symptome bei } \\
\text { Beteiligung der hinteren } \\
\text { Schädelgrube }\end{array}$ & $\begin{array}{l}\text { Internukleäre } \\
\text { Ophthalmoplegie }\end{array}$ & Ataxie & $\begin{array}{l}\text { Area postrema Syndrom } \\
\text { mit Singultus, Übelkeit } \\
\text { und Erbrechen }\end{array}$ \\
\hline ADEM & selten & $\begin{array}{l}\text { Häufig, insbesondere } \\
\text { bei jungen Kindern }\end{array}$ & gelegentlich \\
\hline \multicolumn{4}{|l|}{ Labor } \\
\hline Oligoklonale Banden (Liquor) & $>80 \%$ & Etwa $10 \%$ & Etwa $10 \%$ \\
\hline Serum Anti-MOG Ak & $<5 \%$ (niedriger Titer) & $100 \%$ & $0 \%$ \\
\hline Serum Anti-AQP4 Ak & $0 \%$ & $0 \%$ & $100 \%$ \\
\hline Pleozyotose (Liquor) & $<50 \%$ & $>70 \%$ & $>70 \%$ \\
\hline
\end{tabular}

Tabelle 1: (Modifiziert nach 11): Häufige ADE Präsentationen und assoziierte Merkmale bei Multipler Sklerose, MOGAD und AQP4-NMOSD

herige Abgrenzung von MOGAD und AQP4-NMOSD ist elementar, da bei Letzteren eine Verschlechterung unter einigen der für die MS-Therapie zugelassenen Substanzen auftreten kann.

In der Langzeit-Therapie der pädiatrischen MS unterscheidet man Immunmodulation von (selektiver) Immunsuppression. Bei der Immunmodulation werden beispielsweise die Verhältnisse der Th1 zu den Th2 Zellen medikamentös verschoben, der Patient ist aber nicht im klassischen Sinne immunsupprimiert. Diese Form der Therapie hat den Vorteil, dass sie über längere Zeit ohne relevante Risiken durchgeführt werden kann. Der Nachteil ist die oftmals unzureichende Wirksamkeit («Break-through disease»), vor allem früh im Krankheitsverlauf. Zudem sind diese Präparate nur als Injektionspräparate verfügbar, was per se für das Kindes- und Jugendalter nicht optimal ist. Beide immunmodulatorischen Substanzklassen (Glatirameracetat und Interferone) sind von der Swissmedic für die Therapie der pädiatrischen MS zugelassen. Die Zulassung basiert auf Daten mehrerer Beobachtungsstudien. Bei der (selektiven) Immunsuppression werden gezielt einzelne Kompartimente des Immunsystems ausgeschaltet. Dies geschieht durch Sequestrierung (Fingolimod als orale Form), Blockade an der Blut-HirnSchranke (Natalizumab als monatliche Infusion) oder gezielte Elimination einzelner Subpopulationen (anti-CD20-Therapie als halbjährliche Infusion). Diese Therapieformen sind potenter, gleichzeitig aber auch risikobehafteter betreffend potentieller Langzeitnebenwirkungen. Allerdings lässt sich die Krankheit in den ersten Jahren oftmals erst durch eine immunsuppressive Therapie erfolgreich kontrollieren. In diesen Substanzklassen ist derzeit einzig Fingolimod von der Swissmedic als first-line Therapie zugelassen, die übrigen Präparate werden bei Bedarf und nach sorgfältiger Risiko-Nutzen-Abwägung off-label eingesetzt. Die Zulassung von Fingolimod, einem Sphingosin1-Phosphat-Rezeptor Blocker, stellt einen Meilenstein in der Behandlung der pädiatrischen MS dar, da es das erste Präparat ist, dessen Wirksamkeit und Verträglichkeit im Rahmen einer randomisiert kontrollierten Studie mit double-dummy design (PARADIGMS-Studie) erfolgreich bei Kindern und Jugendlichen mit MS bewiesen worden is $t^{13}$. Bei Natalizumab, einem a4ß1-Integrin Blocker, ist aus grossen Fallserien bekannt, dass es bei Adoleszenten äusserst wirksam und gut verträglich ist ${ }^{14)}$. Da mittlerweile diverse Alternativen bei hoch aktiver MS zur Verfügung stehen, sollte sich der Einsatz von Natalizumab auf JC-Virus negative Patienten beschränken, welche diesbezüglich standardisiert monitorisiert werden. Bei Rituximab, einem monoklonalen Antikörper, der zu einer transienten CD20-Depletion führt, besteht der Vorteil, dass es gewichtsadaptiert verabreicht und somit auch bei jüngeren MS Patienten zum Einsatz kommen kann. Zudem ist Rituximab in der Kinderheilkunde nicht unbekannt und stellt daher kein «neues» Präparat in dieser Altersgruppe dar. Weitere neue Präparate, die die Zulassung für die Therapie MS bei Erwachsenen bereits besitzen, sollen im Rahmen pädiatrischer Studien evaluiert werden. 


\section{Fortbildung}

Aufgrund der soeben ausgeführten Komplexität werden in der internationalen wissenschaftlichen Literatur derzeit zwei Therapieansätze für die Langzeittherapie bei pädiatrischer MS diskutiert:

Start slow and escalate: Bei diesem Therapieansatz wird in der Regel mit einem immunmodulierenden Präparat (Interferon oder Glatirameracetat) begonnen und erst bei inadäquatem Ansprechen auf ein immunsuppressives Präparat eskaliert. Der Vorteil dieses Therapieansatzes besteht darin, dass die Therapie über Jahre ohne Risiko schwerwiegender Nebenwirkungen durchgeführt werden kann. In der Realität sprechen leider nur wenige Patienten zufriedenstellend auf diese Präparate an und die Therapieeskalation ist häufig unvermeidbar.

Start strong, maintain remission: Dieser Therapieansatz basiert auf der hohen Krankheitsaktivität, die früh von kognitiven Einschränkungen und Hirnatrophie begleitet ist. Mit dieser Therapiestrategie wird das Ziel verfolgt, die Krankheitsaktivität der MS schnellstmöglich zu kontrollieren. Hierfür kommt gleich zu Beginn ein immunsuppressives Präparat zum Einsatz. Bei anhaltender Stabilität kann eine Deeskalation erwogen werden. Eine rasche Kontrolle der Krankheitsaktivität und Rückkehr in den normalen Alltag sowie gute Verträglichkeit der Präparate sind Vorteile, das Abwägen des Nutzen-Risiko-Verhältnisses

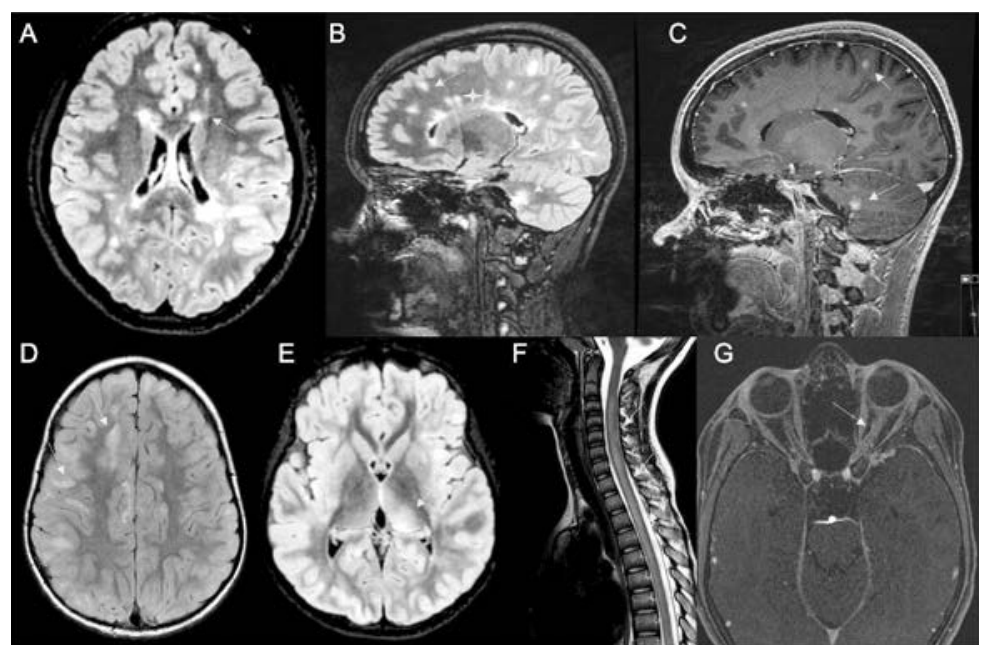

Abbildung 1: A-C MRI einer 15-jährigen Jugendlichen mit Multipler Sklerose. FLAIR ( $A, B)$ : Disseminierte Läsionen periventrikulär («Dawson-Finger»), Stern), juxtakortikal und infratentoriell, T1 (C): Kontrastmittel-Aufnahme einiger Läsionen. D-F MRI eines 5-jährigen Mädchens mit ADEM. T2 (D), FLAIR (E): Unscharf begrenzte, flaue kortikale, subkortikale und thalamische Läsionen, T2 (F): Transverse Myelitis, langstreckig. G MRI eines 10-jährigen Mädchens mit Optikusneuritis (T1 mit KM): Schwellung und Kontrastmittelaufnahme des linken $N$. opticus und der Zeitpunkt der Deeskalation die Herausforderungen dieses Therapieansatzes.

Welcher der beiden Therapieansätze beim jeweiligen Patienten zum Einsatz kommt, muss individuell entschieden werden. Die Therapieempfehlung wird durch Alter des Patienten, Krankheitsaktivität und Schweregrad bei Diagnosestellung, Erholung vom ersten Schub und mögliche Begleiterkrankungen (selten im Kindes- und Jugendalter) massgeblich beeinflusst. Da eine Langzeittherapie letztendlich nur mit der Einsicht in die Therapienotwendigkeit des Jugendlichen über längere Zeit erfolgreich möglich ist, ist auch der Wunsch des jungen Patienten in der Wahl des MS-Therapeutikums mit zu berücksichtigen.

Von den «natural history studies» ist bekannt, dass die Konversion in die sekundär progrediente Phase im jungen Erwachsenenalter von der initialen Krankheitsaktivität abhängig ist ${ }^{3}$. Ferner konnten neuroradiologische Studien zeigen, dass die im MRI als «normal appearing white matter» alles andere als normal is ${ }^{15)}$. Die Schlussfolgerung «nicht behandeln kommt nicht gut» ist wissenschaftlich ausreichend belegt. Daher ist bei allen Kindern und Jugendlichen mit einer MS Diagnose eine immunmodulierende oder immunsuppressive Langzeittherapie empfohlen.

\section{Zusammenfassung}

MS im Kindes- und Jugendalter ist selten und zeigt sich klinisch meist mit einem subakuten fokal-neurologischen Defizit, welches mit typischen Befunden im MRI des zentralen Nervensystems einhergeht. Differentialdiagnostisch steht die Abgrenzung zu antikörpervermittelten entzündlichen Hirnerkrankungen wie MOGAD oder NMOSD im Vordergrund; insbesondere beim jüngeren Kind sollte auch an angeborene Stoffwechselstörungen, die die weisse Substanz betreffen, gedacht werden. Eine MS im Kindes- und Jugendalter verläuft schubförmig und gehört zu den chronisch-entzündlichen ZNS Erkrankungen. Die chronisch-entzündliche Aktivität im sich noch entwickelnden Gehirn wird aktuell als Hauptursache für die frühen kognitiven Probleme angesehen, die diese jungen Patienten zeigen. Therapeutisch stehen mittlerweile sehr effektive und gut verträgliche Immunmodulatoren bzw. Immunsuppressiva zur Verfügung. Die Auswahl des geeigneten Therapeutikums bleibt ein individueller Entscheid und gehört in die Hände von Spezialisten, die über ausreichend Erfahrung in der Diagnose und Therapie dieses Krankheitsbildes im Kindesalter verfügen. (Abbildung 1)

Für das Literaturverzeichnis verweisen wir auf unsere Online Version des Artikels.

\footnotetext{
Autorinnen

PD Dr. med. Sandra Bigi, Institut für Sozial- und Präventivmedizin, Universität Bern und Universitätsklinik für Neurologie, Inselspital, Bern

Dr. med. Annette Hackenberg, Abteilung Neuropädiatrie, Universitäts-Kinderspital Zürich Eleonorenstiftung, Zürich

Die Autorinnen haben keine finanziellen oder persönlichen Verbindungen im Zusammenhang mit diesem Beitrag deklariert.
} 